\title{
Epidemiologia da Cefaléia em Crianças e Adolescentes
}

\author{
Liselotte Menke Barea*, Newra Tellechea Rotta** \& \\ Fernando Gustavo Stelzer ${ }^{* \star *}$
}

\begin{abstract}
RESUMO
Cefaléia é uma queixa comum entre crianças $e$ adolescentes, com altos indices de prevalência nesse grupo etário, onde se evidencia predisposiçāo familiar e predominância no sexo feminino após a adolescência. Os estudos epidemiológicos de cefaléia têm sido comprometidos por problemas metodológicos, sobretudo porque o diagnóstico baseia-se quase que exclusivamente na experiência clínica. Dessa forma ocorrem na literatura um grande número de incongruências relacionado à epidemiologia descritiva, à caracterizaçāo de subtipos e à identificação dos fatores de risco para cefaléia. $O$ objetivo deste trabalho é revisar dados epidemiológicos de cefaléia e dos subtipos mais freqüentes entre crianças e adolescentes e os fatores risco associados.
\end{abstract}

\section{UNITERMOS}

Epidemiologia, cefaléia, crianças, enxaqueca, cefaleia tensional.

\footnotetext{
Professor Adjunto - Doutor em Neurofarmacologia. Professor Adjunto de Neurologia da Fundação Faculdade Federal de Ciências Médicas de Porto Alegre (FFFCMPA).

** Professor Adjunto - Livre-Docente em Neurologia. Professor Adjunto de Pediatria da Universidade Federal do Rio Grande do Sul.

*** Acadêmico da FFFCMPA.
}

\section{INTRODUÇÃO}

Cefaléia é um dos sintomas mais comuns e complexos na clínica médica. É uma situação de grande morbidade, pois nos casos em que se apresenta de forma intensa e freqüente provoca repercussões sociais e econômicas importantes. Além de interferir na vida cotidiana dos indivíduos afetados, a cefaléia exerce também efeitos adversos sobre a produtividade ${ }^{30,1}$.

A despeito de sua importância e frequência, a cefaléia passou a ser identificada como sintoma relevante na patologia pediátrica em 1873 , quando o pediatra britânico William Day dedicou um capítulo de seu livro ao tema cefaléia.

\section{Revisão dos estudos epidemiológicos em cefaléia em crianças e adolescentes}

Vahlquist \& Hackzel, em 1949, com o reconhecimento da enxaqueca infantil como entidade nosológica, estabeleceram um marco no estudo da cefaléia na infância. Bille, em $1962^{6}$, realizou o primeiro estudo epidemiológico no qual os pais de 9.059 escolares suecos responderam um questionário sobre cefaléia demonstrando que, até a idade de 7 anos, $40 \%$ das crianças já havia experimentado esse sintoma e, até os 15 anos, $75 \%$ dessa população já o havia referido. Quanto ao tipo de cefaléia, Bille" observou que a prevalência de enxaqueca aumentava progressivamente com a idade: de $1,4 \%$ aos 7 anos para $5,3 \%$ aos 15 anos. Na idade pré-puberal não havia diferença quanto ao sexo, sendo a predominância no sexo feminino assinalada após os 13 anos.

Dalsgaard-Nielsen \& Ulrich (1973) ${ }^{8}$ relataram uma prevalência crescente de enxaqueca por grupo etário de crianças dinamarquesas, ou seja, de $3 \%$ aos 7 anos e de $9 \%$ aos 15 anos; a prevalência na vida adulta para o sexo masculino permaneceu em torno de $11 \%$, enquanto que para as mulheres alcançou índices de $19 \%$ aos 40 anos de idade. 
Desde então tem havido um crescente interesse dos estudiosos do assunto em estabelecer a prevalência do sintoma cefaléia na população infanto-juvenil.

Deubner $(1977)^{9}$, numa amostra de 600 escolares de 10 a 20 anos, do País de Gales, utilizando entrevista individual e parental em subamostras, observou uma prevalência de cefaléia no último ano de $78 \%$. Enxaqueca foi encontrada em $18,5 \%$ da amostra, havendo predominância no sexo feminino, não variando significativamente com a idade e classe social. O autor assinala a maior sensibilidade da entrevista com o paciente em relação aos pais.

Egermark-Eriksson $(1982)^{11}$ aplicou um questionário sobre cefaléia em uma amostra de 402 indivíduos não-representativa da população escolar sueca. Referiram uma prevalência de cefaléia de $75 \%$ que diferia significativamente de acordo com o sexo e a idade, predominando no sexo feminino e na idade acima de 11 anos.

A prevalência de cefaléia no último ano observada entre 3.784 escolares finlandeses com idade de 13 anos foi de $82 \%{ }^{3(1)}$. Entre os alunos que autopreencheram o questionário, constatou-se que as meninas tinham cefaléias mais frequientemente e que enxaqueca ocorreu em $11,3 \%$ dos escolares.

Numa amostra aleatória por conglomerado de cerca de 2.300 escolares holandeses de 10 a 17 anos, $88 \%$ dos estudantes referiram através do questionário de Waters ter apresentado cefaléia no último ano, sendo mais frequiente no sexo feminino ${ }^{21}$.

Através de entrevista telefônica de cerca de 10.000 pessoas entre 12 e 29 anos de idade nos EUA, Linet e cols. ${ }^{17}$ observaram que $57,1 \%$ no sexo masculino e $76,5 \%$ no feminino tinham referido cefaléia no mês prévio à entrevista, sendo que a taxa de freqüência para enxaqueca no mesmo período foi de $3 \%$ e $7,4 \%$, respectivamente.

Brattberg (1994) ${ }^{7}$ estudou a incidência de lombalgia e cefaléia em uma amostra de 1245 escolares com idades entre 8 a 17 anos na Suécia. Na população estudada, $28 \%$ apresentaram lombalgia e $48 \%$ cefaléia. Em todas as faixas etárias, os sintomas e sua recorrência foram mais prevalentes entre as meninas. Houve associaçāo estatisticamente significativa entre fatores emocionais, sociais e psicológicos e os sintomas referidos. Não houve relação entre as queixas e os fatores físicos.

As discrepâncias entre estimativas de prevalência de cefaléia são devidas aos diferentes critérios de seleção populacional adotados pelos diversos autores. São numerosos os estudos de prevalência em grupos seletivos de pacientes como serviços de emergência, hospitais, clínicas e diferentes grupos ocupacio- nais ${ }^{10,19,12}$, enquanto estudos na população são mais escassos 3 3i.1.5.

Outro aspecto que interfere na avaliação dos estudos de prevalência de cefaléia ć a ausência de marcador biológico. Diversos neurotransmissores têm sido implicados na gênese da cefaléia e enxaqueca desde as observações de Sicuteri e cols. $(1961)^{29}$, em que " principal metabólito urinário da serotonina, 0 ácido 5 hidroxindolacético, estava aumentado durante as crises enxaquecosas. Essas observações foram confirmadas c ampliadas ao longo dos anos por diversos autores que através de estudos farmacológicos sugeriram que at eficícia de várias drogas antienxaquecosas estava relacionada com a interaçĩo a nível de subtipos de receptores de vários neurotransmissores e, em especial, da serotonina ${ }^{22,4}$. Apesar dessas evidências, nāo existe marcador biológico para cefaléia ou enxaquecil, o que dificulta a realização e avaliação de estudos epidemiológicos nessa área.

Até o momento, a maioria dos estudos desenvolvidos basearam-se em critérios diagnósticos vagos, amplos e que permitiam interpretação pessoal. Em 1962, o "Adl Hoc Committee on Classification of Headaches" propôs uma classificação que dividia as cefaléias em "vasculares tipo enxaqueca", "vasculares não-enxaquecosas", "cefaléia por contração muscular", "cefaléias combinadas", entre outras. Esse sistema de classificação possuía critérios vagos e ambíguos, de baixa sensibilidade e especificidade diagnóstica, o que impedia a comparaçāo entre estudos, além de ter utilidade praticamente nula quando aplicados a crianças e adolescentes. Esse fato motivou alguns autores a propor outros critérios para o diagnóstico de enxaqueca na infância.

Duas definições alcançaram grande repercussão na literatura sobre enxaqueca na infância. Vahlquist, em $1955^{32}$, definiu-a como cefaléia episódica separada por intervalos livres de sintomas associada a 2 ou até 4 dos seguintes caracteres: aura visual no início da crise; náuseas ou vômitos; unilateralidade da dor e história familiar positiva. Prensky, em 1976 ${ }^{23}$, sugeriu como critérios diagnósticos a presença de cefaléia episódica intervalada por ausências de sintomas e acompanhada de no mínimo 3 das seguintes situações: aura, náuseas ou vômitos, unilateralidade, história familiar positiva, dor pulsátil e alívio com o sono.

A partir do novo sistema de classificação, desenvolvido pela "International Headache Society" (IHS) em 1988, pode-se dispor de critérios diagnósticos operacionais para os distúrbios cefálicos ${ }^{13}$. Esse sistema classificatório tem permitido uma maior uniformidade diagnóstica, ampliando a possibilidade de comparaçōes entre os diferentes estudos. 
São, no entanto, escassos os trabalhos de prevalência publicados usando esses critérios. O primeiro estudo foi realizado por Rasmussen e cols. ${ }^{26}$, numa amostra de 1.000 homens e mulheres, de 25 a 64 anos, da cidade de Copenhague. Encontraram uma prevalência, ao longo da vida, de cefaléia em $96 \%$, de enxaqueca em $16 \%$ e de cefaléia tipo tensional em $78 \%$ dos entrevistados. Stewart e cols. ${ }^{31}$ publicaram seu estudo baseado numa amostra de 20.468 sujeitos, que responderam a um questionário através do qual os autores estabeleceram a prevalência de uma ou mais crises de enxaqueca por ano em $17,6 \%$ das mulheres e em $5,7 \%$ dos homens dos EUA. No que se refere à população infantil, Kristjánsdóttir \& Wahlberg ${ }^{15}$ evidenciaram uma prevalência semanal de cefaléia de $21,9 \%$ significativamente maior entre meninas na faixa etária de 11 a 12 anos e de nível socioeconômico inferior, entre 2.400 escolares islandeses que autopreencheram um questionário. Em Aberdeen, na Escócia, Abu-Arefeh e Russel', entrevistando $10 \%$ da população escolar de 5 a 15 anos, estimaram taxas de prevalência de $66 \%$ de cefaléia no último ano, sendo $22 \%$ severas a ponto de interferir nas atividades rotineiras; dessas, $10,6 \%$ eram cefaléia do tipo enxaqueca e $0,9 \%$ do tipo tensional. A prevalência de enxaqueca aumentou com a idade bem como a preponderância no sexo feminino. Nessa população, esses autores, em 1995, compararam a prevalência e os achados clínicos de enxaqueca abdominal e de cefaléia enxaquecosa segundo os critérios diagnósticos da IHS, encontrando prevalência de $10,6 \%$ de enxaqueca e $4,1 \%$ de enxaqueca abdominal. As crianças com enxaqueca abdominal apresentaram características sociodemográficas, padrão de recorrência dos sintomas, fatores desencadeantes e de alívio e sintomas associados semelhantes às portadoras de cefaléia enxaquecosa. Os autores sugerem, baseados em suas similaridades, que as duas entidades possam ter patogênese comum. Com base nos critérios diagnósticos parat enxaqueca da IHS, Raieli e cols. ${ }^{24}$ realizaram um estudo epidemiológico em uma população escolar com idades entre 11 a 14 anos, na Itália. A prevalência de enxaqueca sem aura foi de $2,35 \%$, enquanto que enxaqueca com aura teve prevalência de $0,62 \%$ na amostra estudada. Enxaqueca sem aura teve distribuição similar entre os sexos e a enxaqueca com aura foi mais prevalente no sexo feminino.

Revisando retrospectivamente 312 pacientes ambulatoriais portadores de cefaléia crônica de uma clínica de neurologia infantil, Nevo e cols. ${ }^{18}$ diagnosticaram enxaqueca em $54 \%$ da amostra e cefaléia tensional em $22 \%$. A maioria dos casos de enxaqueca foram classificados com enxaqueca sem aura, sendo que enxaqueca com aura foi encontrada em $8,8 \%$ dos casos e enxaqueca complicada em $5,3 \%$. Cefaléia de curta duração, durando de segundos a poucos minutos, loi encontrada em $5,1 \%$ das crianças, sendo que nesse subgrupo foi encontrada grande freqüência de atividade epileptiforme no EEG.

Registra-se uma carência acentuada de investigações epidemiológicas sobre síndromes cefálicas em países não-industrializados. Arregui e cols. ${ }^{3}$, usando os critérios da IHS; estabeleceram a prevalência de enxaqueca no Peru que variou de $3,6 \%$ ao nível do mar até $12,4 \%$ nos Andes, enquanto cefaléia tensional ocorreu em $9,5 \%$ em ambos os locais.

Na literatura brasileira encontram-se alguns estudos observacionais descritivos em grupos seletivos de pacientes. Rotta e cols. ${ }^{27}$ observaram nítida prevalência de cefaléias vasculares episódicas tipo enxaqueca, $58,4 \%$, sobre as cefaléias tensionais, $20,8 \%$, sem predominância de sexo, entre 48 pacientes, de 5 a 16 anos, provenientes de clínica especializada. Já em 1985 , Rotta e cols. ${ }^{28}$ com o objetivo de estimar a prevalência do sintoma cefaléia no contexto global da patología pediátrica a nível hospitalar, realizaram um estudo retrospectivo no qual entre 8.284 consultas em crianças de um a quinze anos de idade, a queixa cefaléia foi encontrada em $5,2 \%$ dos casos; quanto à natureza da cefaléia, o maior contingente ficou com as cefaléias agudas, de origem extracraniana, representadas na sua maioria pelas infecçōes de vias aéreas superiores. Silva (1985), apesar de referir-se ao indicador epidemiológico incidência, estimou a frequiência de síndromes cefálicas em clínica especializada, num desenho experimental incompleto tipo caso-controle. Um estudo observacional, com delineamento transversal, foi realizado por Barea e cols. ${ }^{5}$, tendo sido estimada al prevalência de cefaléias ao longo da vida de $93,3 \%$, no último ano de $82,9 \%$, na semana de $31,4 \%$ e no dial de $8,9 \%$ em uma amostra de 538 sujeitos de 10 a 18 anos da rede escolat de Porto Alegre. A prevalência de cefalćia no último ano demonstrou estar associada positivamente ao sexo e idade. Nessa amostra, segundo os critérios operacionais da IHS, a enxacueca foi diagnosticada em $9,9 \%$ e a cefaléia tipo tensional em $77,6 \%$.

$\mathrm{Na}$ atualidade, os pesquisadores dessa área têm se preocupado em validar os critérios diagnósticos para as cefaléias, especialmente a enxaqueca, na população infanto-juvenil.

Wöber-Bingol e cols. ${ }^{34}$ analisaram os critérios de cefaléia propostos pela IHS comparando-os com os de Vahlquist $^{32}$, em uma população de 437 crianças e adolescentes de uma clínica especializada em cefaléia. Encontraram boa concordância entre os dois critérios 
acima, no diagnóstico do tipo de cefaléia, concluindo ser a classificação da IHS útil em crianças e adolescentes. Ressaltam, no entanto, a limitação de seu uso em estudos populacionais. Os autores propõem alteraçōes nos critérios diagnósticos de enxaqueca em pacientes com idade inferior a 15 anos, com redução da duração da dor de 2 para 1 hora, bem como inclusão da qualificação da severidade dos sintomas acompanhantes no diagnóstico de enxaqueca e cefaléia tensional.

Winner e cols. ${ }^{33}$ propuseram modificações (IHS-R) dos critérios de enxaqueca estabelecidos pela IHS, com o objetivo de aumentar a sensibilidade no diagnóstico de enxaqueca na população pediátrica, com idade inferior a 15 anos. Os critérios diagnósticos para enxagueca em crianças e adolescentes foram revisados com especial atenção em relação à duração da dor, localização, intensidade e sintomas relacionados à fono e fotofobias. Para enxagueca sem aura, os autores propõem uma diminuição do tempo mínimo de duração da dor de 2 horas para 30 minutos, além da localização da dor poder ser uni ou bilateral (frontal/temporal). Em relação à enxaqueca com aura, a única alteração proposta é a redução da duração da dor de 2 horas para 30 minutos. Os autores estudaram retrospectivamente uma população de 45 crianças comparando os critérios da IHS, da IHS-R e os de Vahlquist ${ }^{32}$. A IHS-R mostrou maior sensibilidade com maiores taxas de diagnóstico em relação aos outros dois critérios analisados.

Os pesquisadores têm encontrado dificuldade em estabelecer critérios diagnósticos para a enxaqueca na criança que sejam ao mesmo tempo sensíveis e específicos. A enxaqueca na infância difere em alguns aspectos daquela no adulto. Alguns critérios, sobretudo aqueles relacionados ao caráter da dor, para diagnóstico de enxaqueca são de difícil compreensão para a populaçāo pediátrica, principalmente, para as crianças de menor idade.

\section{CONSIDERAÇÕES FINAIS}

Existem muitas questões ainda não esclarecidas no que se refere à epidemiologia, fisiopatologia e clínica das cefaléias, as quais são indissociáveis na sua abordagem.

Porém parece haver um consenso na literatura no que se refere aos altos índices de prevalência desse sintoma na população infanto-juvenil, bem como dos subtipos enxaqueca e cefaléia tensional. As diferenças de prevalência obtidas a favor do sexo feminino a partir da adolescência têm sido constantemente observadas, bem como a predisposição familiar.
A despeito das restrições aos critérios diagnósticos para cefaléia enxaquecosa e tensional nessa faixal etária, com dados disponíveis nesta revisão relativos a magnitude populacional, pode-se concluir pela importância desse sintoma como motivo de consultas médicas e prejuízos pessoais e sociais.

Deve-se enfatizar que as estimativas de prevalência dos distúrbios cefálicos são instrumentos informativos para programas de saúde, os quais, à luz dos dados, devem objetivar aos clínicos orientação paral adequalo diagnóstico, tratamento e controle dos fattores de riseo nos pacientes expostos sempre que possivel.

\section{SUMMARY}

Headache is a common disorder among children and adolescents for whose high prevalence is identified. There is a higher female prevalence alter adolescence and familiar predisposition.

The study of headache types and associated risk factors has been hampered by a number of methodological problems, specially the diagnosis is based almost entirely on clinical experience. As a result of these problems, a number of inconsitencies in descriptive epidemiology of headache, the characterisation of the subtypes, and the identification of risk factors are apparent in the literature.

The purpose of this paper is to provide a review of the descriptive epidemiology of headache and headache subtypes among chitdren and adolescents and a summary overview of associated risk factors

\section{KEY WORDS}

Epidemiology, headache, children, migraine, tension-type.

\section{Referências}

1. Abu-Arefeh, I.; Russel, G. Prevalence of headache and migraine in schoolchildren. Br. Med. J., 309: 765-769, 1994.

2. Abu-Arafeh, I.; Russel, G. Prevalence and clinical features of abdominal migraine compared with those of migraine headache. Arch. Dis. Child., 72(5): 413-7, 1995.

3. Arregui, A.; Cabrera, J.; Leon-Verlade, F.; Parades, S.; Viscara, D.; Arbaiza, D. High prevalence of migraine in high altitude population. Neurology, 41: 1668-1670, 1991.

4. Barea, L.M. Efeitos serotonérgicos dos antagonistas dos canais de cálcio, flunarizina e nifedipina. Possivel relação com a atividade antienxaquecosa. Porto Alegre: 1992, 122 Dissertação (Mestrado) - Fundação Faculdade Federal de Ciencias Médicas de Porto Alegre.

5. Barea, L.M. Prevalência de cefaléia e do consumo de analgésicos em escolares de 5 a à 8 a séries de Porto Alegre. Porto Alegre, 1995, 107p. Tese (Doutorado) - Fundaçāo Faculdade Federal de Ciências Médicas de Porto Alegre.

6. Bille, B.S. Migraine in school children. Acta Paediatr. Scand., 51(suppl 136): 1-151, 1962.

7. Brattberg, $G$. The incidence of back pain and headache among Swedish school children. Qual. Life Res., 3 (Suppl) 1:27-31, 1994

8. Dalsgaard-Nielsen, T.; Ulrich, J. Prevalence of heredity of migraine and migranoid headache among 461 danish children. Headache, 12: 168-72, 1973.

9. Deubner, D.C. An epidemiologic study of migraine and headache in 10-20 year olds. Headache, 17: 173-180, 1977.

10. Dhopesh, V.; Anwar, R.; Herring, C.A. Retrospective assessment of emergency department with complain of headache. Headache, 19: 37-42, 1979. 
11. Egermark-Eriksson, I. Prevalence of headache in Swedish schoolchildren. A questionnaire survey. Acta Paediatr. Scand., 71: 135-140, 1982.

12. Forgays, D.G.; Rzewnick. I.R.; Ober, A.J., Forgays, D.K. Headache in college students: a comparison of four populations. Headache, 33: 182-190, 1993.

13. Headache Classification Committee of the International Headache Society. Classification and diagnostic criteria for headache disorders, cranial neuralgias and facial pain. Cephalalgia, 8(suppl.7): 95, 1988.

14. Honkasalo, M.L.; Kaprio, J.; Heikkila, K.; Sillampaa, M.; Koskenvou, $M$. Population based survey of headache and migraine in 22,809 adults. Headache, 13: 403-12, 1993.

15. Kristiánsdóttir, G.; Wahlberg, V. Sociodemographic differences in the prevalence of self-reported headache in Iceland SchoolChildren. Headache, 33: 376-380, 1993.

16. Linet, M.S.; Stewart, W.F. Migraine headache: epidemiologic perspectives. Epidemiol. Rev., 6: 107-139, 1984.

17. Linet, M.S.; Stewart, W.F.; Celentano, D.D.; Ziegler, D; Sprecher, M. An epidemiologic study of headache among adolescents and young adults. J.A.M.A., 261: 2211-2216, 1989.

18. Nevo, Y.; Kramer, U.; Rieder-Groswasser, I.; Harel, S. Clincal caracterization of 312 children with chronic headache. Brain Dev, 16(6): 441-444, 1994.

19. Oguyemi, A.O. Prevalence of headahce among Nigerian university students. Headache, 24: 127-130, 1984.

20. Passchier,J.; Orlebeke,J.F. Headache and stress in schoolchildren: an epidemiological study. Cephalalgia, 5: $167-$ 176, 1985.

21. Passchier,J.; Hunfeld, J.A.M.; Jelicic, M.; Verhage, F. Suggestibility and headache reports in schoolchildren: a problem in epidemiology. Headache, 33: 73-75, 1993.

22. Peroutka, S. J. Antimigraine drug interactions with serotonin receptors subtypes in human brain. Ann. Neurol., 23: 500 $504,1988$.
23. Prensky, A.L. Migraine variants in pediatric patients. Pediat Clin North Am, 23: 461-71, 1976.

24. Raieli, V.; Raimondo, D.; Cammalleri, R.; Camarda, R. Migraine headaches in adolescents: a student populationbased study in Monreale. Cephalalgia, 15(1): 5-12, 1995.

25. Rasmussen, B.K.; Jensen, R.; Schroll, M.; Olesen, J. Epidemiology of headache in a general population - a prevalence study. J.Clin. Epidemiol., 44: 1147-1157, 1991.

26. Rasmussen, B.K.; Olesen, J. Migraine with aura and migraine without aura: an epidemiological study. Cephalalgia, 12: 221 . $228,1992$.

27. Rotta, N.T.; Barea, L.M.; Guardiola, A.; Vallandro, C.; Merúvia M.; Farias, A.S. Cefaléia na infância. Revista da AMRIGS 27: 357-365, 1983.

28. Rotta, N.T.; Lago, I.S.; Ohlweiller, L.; Drachler, M.L.; Souto, L.D.; Lyra, A.B.; Leite, N.D.; Freire, M.E.N. Cefaléia como queixa ambulatorial em pediatria. Revista do Hospital de Clínicas de Porto Alegre, 5: 25-27, 1985.

29. Sicuteri, F.: Testi, H.; Anselmi, B. Biochemical investigation in headache: increase in 5-hidroxyindolacetic acid excretion during migraine. Int Arch Allergy, 19: 55-58, 1961.

30. Sillampää, M. Prevalence of headache in pre-puberty. Headache, 23: 10-14, 1983

31. Stewart, W.F.; Lipton, R.B.; Celentano, D.D.; Reed, M.L. Prevalence of migraine headache in the United States. J.A.M.A., 267: 649-, 1992.

32. Vahiquist, B. Migraine in children. Int.Arch.Allergy, 7: 348355, 1955.

33. Winner, P.; Martinez, W.; Mate, L.; Bello, L. Classification of pediatric migraine: proposed revisions to the IHS criteria. Headache, 35(7): 407-10, 1995.

34. Wöber-Bingöl, C.; Wōber,C.; Kaurwautz, A.; Vesely, C. Wagner-Ennsgraber, C.; Amninger, G.P.; Zebenholzer, K.; Geldner, J.; Baischer, W.; Schuch, B. Diagnosis of headache in childhood and adolescence: a study in 437 patientes. Cephalalgia, 15: 13-21, 1995. 